\title{
A "Vase" or A "Face": is (in Part) an Influence of Image Schemas
}

\author{
Mai Sabry Saleh \\ Environmental and Occupational Medicine Department, National Research Center, Dokki, Giza, \\ Egypt \\ nouranomer@gmail.com
}

\begin{abstract}
Perceptual organization concerns the way sensory information is organized and formed into a perceptual object. The role of visual schemas is to match visual sensory inputs with object representations in our memory for its recognition. Both bottom-up and top-down attention mechanisms guide visual schemas. Yet, how this object is rendered meaningful? And does abstract knowledge linked to an object plays a role in its visual perception? The answer for these questions is discussed in the few coming paragraphs.
\end{abstract}

Keywords: perceptual organization; visual schema; image schema; figure-ground segregation

In order to efficiently process visual input, the brain actively uses previous knowledge and expectations about what the world should look like (Jolij \& Meurs, 2011). According to Hochberg (1968) any analysis of shape and scene perception must be conducted on the mental representation or image in the mind's eye which he called a schematic map or a mental schema rather than on the stimulus itself.

In this consequence we can learn more about visual schemas which are defined as active functional units or object representations that cooperate and compete to determine which representation best matches the object (Yuanlong Yu, Mann \& Gosine, 2012). According to Logan (1996) objects refer to perceptual groups or units. These perceptual groups are formed through the application of the wellknown gestalt principles of organization. At the level of its constituting parts, a single perceptual group or object may have a hierarchical organization of its parts previously defined by certain perceptual principles. Vecera \& Behrmann (2001) refer to this perceptual grouping definition of object as a grouped array representation which is an array-format that codes features in specific retinal locations into coherent chunks of visual information that correspond to objects or shapes. Significantly, this group array can introduce a more profound description of visual schemas.

In coherence with group array is perceptual organization as described by Vyvyan Evan (2010). In his consideration a perceptual organization is integration of neural codes by the brain to form a perceptual object which he called a percept. Yet he differentiated in the process of perception between such formation of the percept and the formation of a concept which he denoted by image schema. Then he defined a concept or image schema as past experiences and conceptual knowledge which is meant to identify and categorize the percept. Further he made a comparison between percepts and concepts or let us say visual schemas and image schemas in one crucial aspect of our discussion. This aspect is how do percepts and concepts arise?

Precisely here, lies the point of our interest. visual schemas rely on multimodal sensory inputs where computing and processing take place at the same instance of perception with bottom-up and top-down processing integrating in an interweaving manner applying through their various well known mechanisms. Figure ground segregation - with border ownership as one of its crucial elements - is one example upon which such mechanisms are applied (Mihalas, Dong, von der Heydt, \& Niebur, 2011). On the other hand concepts or image schemas arise from abstract conceptualization where schematization includes a wide range of information types which is not restricted to instantaneous sensory inputs for resolving a certain percept. 
Image schema in my speculation is simulated to a magnificent creature with outstanding abilities in our minds. It is very special and unique for each individual as it is the sum of all the experience of his own life. In my opinion, image schemas can seriously influence by a mean or another our perception, and that is why not how some of us pick up the face while others tend to observe the vase.

\section{REFERENCES}

Evans, V. (2010). The perceptual basis of spatial representation. In V. Evans, \& P. Chilton (Eds.), Language, Cognition and Space: The State of the Art and New Directions (pp. 21-47). England \& Wales: Equinox Publishing.

Hochberg, J. (1968). In the mind's eye. In R. N. Haber (Ed.), Contemporary theory and research in visual perception (pp. 309-331). NY: Holt Rhinehart and Winston.

Jolij, J., \& Meurs, M. (2011). Music Alters Visual Perception. PLoS ONE, 6(4): e18861.

Logan, G. D. (1996). The CODE theory of visual attention: An integration of space-based and objectbased attention. Psychological Review, 103, 603-649.

Mihalas, S., Dong, Y., von der Heydt, R., \& Niebur, E. (2011). Mechanisms of perceptual organization provide auto-zoom and auto-localization for attention to objects. Proc Natl Acad Sci USA, 108(18), 7583-7588.

Vecera, S. P., \& Behrmann, M. (2001). Attention and unit formation: A biased competition account of object-based attention. Advances in Psychology, 130, 145-180.

Yuanlong Yu, Mann, G.K.I., \& Gosine, R.G. (2012). A Goal-Directed Visual Perception System Using Object-Based Top-Down Attention. Autonomous Mental Development, IEEE Transactions, 4(1), 87 - 103.

\section{AUTHOR's BIOGRAPHY}



Mai Sabry Saleh, Ph.D., in biochemistry. Graduated from faculty of Pharmacy, Cairo University. Interested mainly in cognitive science, health and wellbeing, human recourses development and stress detection and management. Has international publications in the aforementioned fields and invented a tool for learning style assessment; Intellectual Style Inventory (ISI). A multisystem memory model for conceptual learning ; Integrated Model of Mind (IMM) is another innovation of the author which is a psychological model that overcomes limitations of the latest popular models describing cognition and abstract conceptualization. 